\title{
ABVD und BEACOPP bei HL-Patienten mit hohem Risiko
}

\begin{abstract}
Von welchem Regime profitieren Patienten mit fortgeschrittenem HodgkinLymphom (HL) und schlechter Prognose mehr: Von ABVD8 oder von BEACOPP4+4? Diese Frage sollte der EORTC-Studie 20012 beantwortet werden.
\end{abstract}

0 as Protokoll ABVD (Doxorubicin, Bleomycin, Vinblastin und Dacarbazin) ist bei fortgeschrittenem HodgkinLymphom (HL) Standard, da es sich gegenüber anderen Regimes als wirksamer oder weniger toxisch erwiesen hatte. Allerdings gilt dies nicht für HL-Patienten mit hohem Risiko. Zwischen 2002 und 2010 wurden daher 550 Patienten mit Hochrisiko-HL im Stadium III oder IV für die Studie 20012 der EORTC rekrutiert. Die Patienten wurden randomisiert entweder mit ABVD oder BEACOPP (Bleomycin, Etoposid, Doxorubicin, $\mathrm{Cy}$ clophosphamid, Vincristin, Procarbazin und Prednison) behandelt. Einschlusskriterien waren: unbehandeltes HL, Performancestatus von 0-2 und ein International Prognostic Score (IPS) $\geq 3.74 \%$ der
Patienten hatten ein HL Stadium IV, $81 \%$ B-Symptome und $59 \%$ einen IPS $\geq 4$.

Insgesamt wurden jeweils 8 Zyklen verabreicht. Im Standard-Arm 8 Zyklen ABVD (ABVD8; $\mathrm{n}=275$ ) und im BEACOPP-Arm 4 Zyklen BEACOPP eskaliert, $_{\text {, }}$ gefolgt von 4 Zyklen BEACOPP baseline $_{\text {(BE- }}$ ACOPP4+4; $n=274)$. Primäre Endpunkte waren ereignisfreies Überleben (EFS), Therapieunterbrechungen und das Fehlen von Komplettremissionen (CR) oder nicht bestätigten CR (CRu), Progression, Rezidiv oder Tod. Sekundäre Endpunkte umfassten CR-Rate, Gesamtüberleben (OS), Lebensqualität, Zweitmalignome und krankheitsfreies Überleben (DFS) bei Patienten mit CR oder CRu.

Nach median 3,6 Jahren erreichten in beiden Armen 82,5\% der Patienten eine
CR oder CRu. Nach 4 Jahren waren die Ergebnisse zwischen ABVD8 und BEACOPP4+4 vergleichbar (EFS-Rate: 63,7 vs. 69,3\%, Hazard Ratio [HR] 0,86; $p=0,312$; DFS: 85,8 vs. $91,0 \%$ HR 0,$59 ; \mathrm{p}=0,076$ und OS: 86,7 vs. $90,3 \%$; HR 0,$71 ; \mathrm{p}=$ 0,208). 6 bzw. 5 Patienten starben an den Folgen von Nebenwirkungen, 12 bzw. 26 Patienten brachen die Therapie vorzeitig (vor dem 5. Zyklus) ab, bei 8 bzw. 10 Patienten trat ein Zweitmalignom auf.

Fazit: Beide Regime führten bei Patienten mit fortgeschrittenem Hochrisiko-HL zu vergleichbarem EFS und OS. Zudem war BEACOPP4+4 weder wirksamer noch weniger toxisch als ABVD8.

Kathrin von Kieseritzky

Carde $P$ et al. Eight Cycles of ABVD Versus Four Cycles of BEACOPPescalated Plus Four Cycles of BEACOPPbaseline in Stage III to IV, International Prognostic Score $\geq 3$, High-Risk Hodgkin Lymphoma: First Results of the Phase III EORTC 20012 Intergroup Trial. J Clin Oncol. 2016;34(17):2028-36.

\section{Myelom: Phase-III-Studie mit Ixazomib}

\section{Immunmodulatorische Medikamente und Proteasominhibitoren haben die Therapie des multiplen Myeloms (MM) erheblich verbessert. Nun wurde ein weiterer Proteasominhibitor in einer Phase-III-Studie untersucht.}

$\mathrm{M}$ ehr als 10 Proteasominhibitoren gibt es mittlerweile. Ein weiterer aussichtsreicher neuer Proteasominhibitor ist das oral verfügbare Ixazomib. Es führte in den ersten Studien zu ermutigenden Ergebnissen.

In dieser aktuellen Studie wurden insgesamt 722 Patienten mit rezidiviertem multiplem Myelom behandelt. Sie erhielten randomisiert entweder Ixazomib in Kombination mit Lenalidomid plus Dexamethason (Rd) oder aber ein Placebo plus Rd. Primärer Endpunkt war das progressionsfreie Überleben (PFS).

In der mit Ixazomib/Rd behandelten Gruppe lebten die Patienten mit median 20,6 Monaten gegenüber 14,7 Monaten signifikant länger progressionsfrei als in der Placebo/Rd-Gruppe (Hazard Ratio [HR] 0,74; $p=0,01)$. Der Vorteil der Ixa-
zomib-Addition zeigte sich in allen zu Beginn der Studie definierten Subgruppen. Auch Patienten mit zytogenetischen Hochrisiko-Aberrationen profitierten von der Ixazomib-Kombination.

Sowohl die Gesamtansprechraten als auch komplette bzw. sehr gute partielle Remissionen wurden durch Ixazomib/ Rd verbessert (78 vs. $72 \%$ und 48 vs. $39 \%)$. Zudem sprachen die Patienten rascher (mediane Zeit bis zum Ansprechen: 1,1 vs. 1,9 Monate) und länger (im Median 20,5 vs. 15,0 Monate) auf die Behandlung an.

Nach einer medianen Nachbeobachtungszeit von 23 Monaten war das mediane Gesamtüberleben in keiner Gruppe erreicht. Die Raten schwerer Nebenwirkungen waren in beiden Studienarmen ähnlich (Ixazomib/Rd: $47 \%$, Pla-
cebo/Rd: $49 \%$ ); die Patienten berichteten auch von ähnlicher Lebensqualität. Gestorben sind in der Studienperiode $4 \%$ bzw. $6 \%$ der Patienten.

Die Rate schwerwiegender Nebenwirkungen ( $\geq$ Grad 3) betrug in der Ixazomib/Rd-Gruppe 74\%, in der Placebo/ Rd-Gruppe $69 \%$. Insbesondere Thrombozytopenien vom Grad 3 bzw. 4 waren häufiger (12 bzw. 7 \% vs. 5 bzw. $4 \%$ ). Auch Hautausschläge und gastrointestinale Beschwerden traten unter der IxazomibKombination häufiger auf. Diese waren allerdings vornehmlich von niedrigem Schweregrad. Die Inzidenz peripherer Neuropathie lag bei $27 \%$ im Ixazomib/ Rd- und bei $22 \%$ im Placebo/Rd-Arm.

Fazit: Die Addition von Ixazomib zu Rd war mit einem signifikant längeren PFS verbunden. Zusätzliche toxische Effekte dieses vollständig oralen Regimes waren überschaubar.

Christina Berndt

Moreau P et al. Oral Ixazomib, Lenalidomide, and Dexamethasone for Multiple Myeloma. N Engl J Med. 2016:374(17):1621-34. 